\title{
Energy Harvesting Diamond Channel with Energy Cooperation
}

\author{
Berk Gurakan \\ Sennur Ulukus \\ Department of Electrical and Computer Engineering \\ University of Maryland, College Park, MD 20742 \\ gurakan@umd.edu \\ ulukus@umd.edu
}

\begin{abstract}
We consider the energy harvesting diamond channel, where the source and two relays harvest energy from nature, the relays help deliver the source's messages via signal cooperation, and the source has the option of wirelessly transferring some of its energy to the relays via energy cooperation. We find the optimal offline transmit power allocations and energy transfer policies that maximize the end-to-end throughput. For the case of no energy cooperation, we decompose the problem into inner and outer maximization problems, and solve the overall problem iterating between the two. We show that the class of procrastinating policies, where energy is transferred only when it will be immediately used, is optimal. We then show that the problem with energy cooperation is equivalent to a problem without energy cooperation with suitably modified rate expressions. We show that, in this system, if the source sends more energy to a relay, then it sends less data, showing us how data and energy should flow together optimally in this network.
\end{abstract}

\section{INTRODUCTION}

We consider the energy harvesting diamond channel [1], see Fig. 1, where all transmitters harvest energy from nature. The relays cooperate with the source in the physical layer via signal cooperation [2] and forward the source's data to the destination. In addition, the source cooperates with the relays at the battery level by transferring some of its energy [3] to the relays via energy cooperation [4]. We determine the optimum power allocation and energy transfer policies of the users in order to maximize the end-to-end throughput in this system.

There has been a considerable amount of recent work in power control for energy harvesting communications [5]-[21]. In [5], the transmission completion time minimization problem is solved for an unlimited-sized battery. In [6], the throughput maximization problem is solved and its equivalence to the transmission completion time minimization problem is shown for an arbitrarily sized battery. In [7]-[16], the problem is extended to fading, broadcast, multiple access, interference and relay channels. Throughput maximization problem with battery imperfections is considered in [17], [18] and processing costs are incorporated in [19], [20]. Recently, in [21], a twohop communication system with one and two parallel relays in a half-duplex setting is considered. Energy cooperation is introduced in [4], and jointly optimum power allocation and energy transfer policies are found for one-way [4] and twoway energy cooperation in [22], [23].

This work was supported by NSF Grants CNS 09-64632, CCF 09-64645, CCF 10-18185 and CNS 11-47811.

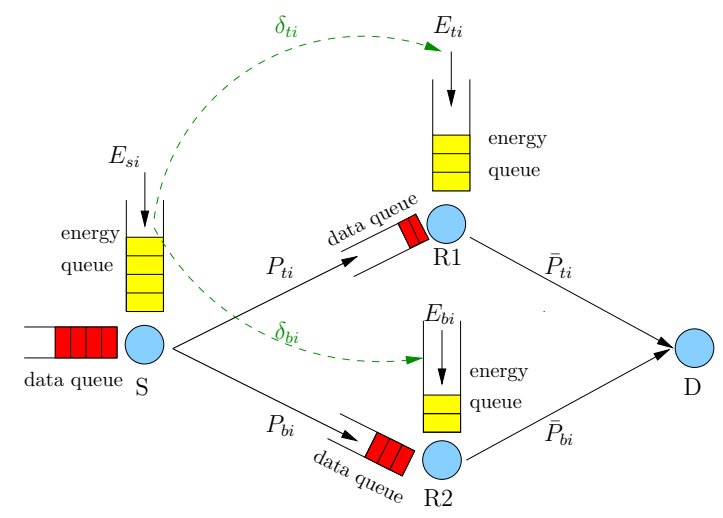

Fig. 1. Energy harvesting diamond channel with energy cooperation.

In this paper, we first consider the case where no energy cooperation is allowed. We decompose the problem into inner and outer optimization problems, which are both convex, and solve the overall problem by iterating between the two, and using directional waterfilling [7]. For the case with energy cooperation, we first show, as in [22], [23], the optimality of procrastinating policies, i.e., that energy must be transferred only when it will be used immediately. Then, we transform the problem with energy cooperation to one with no energy cooperation with suitably modified rate expressions. We show that if energy is transferred to both relays, then the transmit powers of the source to both relays must be equal implying that equal amount of data must be transmitted from the source to the relays; on the other hand, if energy is transferred only to one of the relays then the transmit power of the source to that relay must be less, meaning that if the source sends more energy to a relay then it sends less data. This shows how energy and data flow together optimally in this network.

\section{System Model and Problem Definition}

We consider the energy harvesting diamond channel with energy cooperation shown in Fig. 1. The harvested energies are saved in the corresponding batteries. All of the physical layers are additive white Gaussian noise (AWGN) channels with unit channel gains, and unit AWGN powers. The broadcast link from the source to the relays and the multiple access link from the relays to the destination are orthogonal as in [1]; in addition, in our model, the two broadcast links and the two multiple 
access links are orthogonal among themselves as well. When there is wireless energy transfer, this is done by two separate orthogonal energy transfer units whose coupling frequencies are set differently [3]. Finally, data transmission and energy transfer channels are orthogonal, i.e., energy transfer does not create interference to data communication.

There are $L$ equal length slots. We refer to relay 1 as the top and relay 2 as the bottom relay and use subscripts $t$ and $b$ to denote their parameters; subscript $s$ denotes source node's parameters. In slot $i$, the source, top and bottom relays harvest energy with amounts $E_{s i}, E_{t i}, E_{b i}$. Energy transfer efficiency is $\alpha$ : when the source transfers $\delta_{t i}\left(\delta_{b i}\right)$ amount of energy to the top (bottom) relay, $\alpha \delta_{t i}\left(\alpha \delta_{b i}\right)$ amount of energy enters the energy queue of the top (bottom) relay. We denote the transmission power of the source to the top (bottom) relay as $P_{t i}\left(P_{b i}\right)$, the transmission power of the top (bottom) relay to the destination as $\bar{P}_{t i}\left(\bar{P}_{b i}\right)$. We denote these power and energy transfer sequences with the vectors $\overline{\mathbf{P}}_{t}, \overline{\mathbf{P}}_{b}, \mathbf{P}_{t}, \mathbf{P}_{b}, \boldsymbol{\delta}_{t}, \boldsymbol{\delta}_{b}$.

The energy that has not arrived yet cannot be used, leading to the following energy causality constraints at all transmitters:

$$
\begin{gathered}
\sum_{i=1}^{k} \bar{P}_{t i} \leq \sum_{i=1}^{k}\left(E_{t i}+\alpha \delta_{t i}\right), \quad \forall k \\
\sum_{i=1}^{k} \bar{P}_{b i} \leq \sum_{i=1}^{k}\left(E_{b i}+\alpha \delta_{b i}\right), \quad \forall k \\
\sum_{i=1}^{k} P_{b i}+P_{t i} \leq \sum_{i=1}^{k}\left(E_{s i}-\delta_{t i}-\delta_{b i}\right), \quad \forall k
\end{gathered}
$$

The relays cannot forward data that has not arrived yet, leading to the following data causality constraints at the relays:

$$
\begin{array}{ll}
\sum_{i=1}^{k} \frac{1}{2} \log \left(1+\bar{P}_{t i}\right) \leq \sum_{i=1}^{k} \frac{1}{2} \log \left(1+P_{t i}\right), & \forall k \\
\sum_{i=1}^{k} \frac{1}{2} \log \left(1+\bar{P}_{b i}\right) \leq \sum_{i=1}^{k} \frac{1}{2} \log \left(1+P_{b i}\right), & \forall k
\end{array}
$$

The end-to-end throughput maximization problem subject to energy and data causality constraints is:

$$
\begin{aligned}
\max _{\substack{\bar{P}_{t i}, \bar{P}_{b i}, P_{t i}, P_{b i} \\
\delta_{t i}, \delta_{b i}}} & \sum_{i=1}^{L} \frac{1}{2} \log \left(1+\bar{P}_{t i}\right)+\frac{1}{2} \log \left(1+\bar{P}_{b i}\right) \\
\text { s.t. } & (1)-(5),\left(\overline{\mathbf{P}}_{t}, \overline{\mathbf{P}}_{b}, \mathbf{P}_{t}, \mathbf{P}_{b}, \boldsymbol{\delta}_{t}, \boldsymbol{\delta}_{b}\right) \geq \mathbf{0}
\end{aligned}
$$

The problem in (6) is convex when formulated in terms of the rates as in [4], [21]. However, it is intractable, mostly due to the data causality constraints in (4)-(5). To gain insight, we simplify the problem description by imposing strict delay constraints on the relays, as in [23]. This is tantamount to removing the relay data buffers, and forcing relays to decodeand-forward the data they receive in the same slot. Since there are no data buffers at the relays, there must remain no unforwarded data in any of the slots at both relays, and (4)-(5) should be satisfied with equality for all $k$ :

$$
\begin{array}{ll}
\sum_{i=1}^{k}\left(\frac{1}{2} \log \left(1+P_{t i}\right)-\frac{1}{2} \log \left(1+\bar{P}_{t i}\right)\right)=0, & \forall k \\
\sum_{i=1}^{k}\left(\frac{1}{2} \log \left(1+P_{b i}\right)-\frac{1}{2} \log \left(1+\bar{P}_{b i}\right)\right)=0, & \forall k
\end{array}
$$

which is equivalent to $\bar{P}_{t i}=P_{t i}, \bar{P}_{b i}=P_{b i}, \forall i$. The new system model with strict delay constraints is shown in Fig. 2.

The feasible set can now be written as follows

$$
\begin{aligned}
\mathcal{F}=\left\{\left(\boldsymbol{\delta}_{t}, \boldsymbol{\delta}_{b}, \mathbf{P}_{t}, \mathbf{P}_{b}\right): \sum_{i=1}^{k} P_{t i}\right. & \leq \sum_{i=1}^{k}\left(E_{t i}+\alpha \delta_{t i}\right) \\
\sum_{i=1}^{k} P_{b i} & \leq \sum_{i=1}^{k}\left(E_{b i}+\alpha \delta_{b i}\right) \\
\sum_{i=1}^{k} P_{b i}+P_{t i} & \leq \sum_{i=1}^{k}\left(E_{s i}-\delta_{t i}-\delta_{b i}\right) \\
P_{t k}, P_{b k}, \delta_{t k}, \delta_{b k} & \geq 0, \quad \forall k\}
\end{aligned}
$$

With the strict delay constraints, the problem in (6) becomes:

$$
\begin{aligned}
\max _{P_{t i}, P_{b i}, \delta_{t i}, \delta_{b i}} & \sum_{i=1}^{L} \frac{1}{2} \log \left(1+P_{t i}\right)+\frac{1}{2} \log \left(1+P_{b i}\right) \\
\text { s.t. } & \left(\boldsymbol{\delta}_{t}, \boldsymbol{\delta}_{b}, \mathbf{P}_{t}, \mathbf{P}_{b}\right) \in \mathcal{F}
\end{aligned}
$$

which we solve in the following two sections for the cases of no energy transfer and with energy transfer.

\section{No ENERgy TRAnsfer}

In this case, $\boldsymbol{\delta}_{t}=\boldsymbol{\delta}_{b}=\mathbf{0}$, and the problem becomes:

$$
\begin{aligned}
\max _{P_{t i}, P_{b i} \geq 0} & \sum_{i=1}^{L} \frac{1}{2} \log \left(1+P_{t i}\right)+\frac{1}{2} \log \left(1+P_{b i}\right) \\
\text { s.t. } \quad & \sum_{i=1}^{k} P_{t i} \leq \sum_{i=1}^{k} E_{t i}, \quad \forall k \\
& \sum_{i=1}^{k} P_{b i} \leq \sum_{i=1}^{k} E_{b i}, \quad \forall k \\
& \sum_{i=1}^{k} P_{t i}+P_{b i} \leq \sum_{i=1}^{k} E_{s i}, \quad \forall k
\end{aligned}
$$

We first prove a property of the optimal solution, which states that either the source energy should be fully consumed or the energies at both relays should be fully consumed.

Lemma 1 The optimal power allocations $P_{t i}^{*}, P_{b i}^{*}$ must satisfy either $\sum_{i=1}^{L} P_{t i}^{*}=\sum_{i=1}^{L} E_{t i}$ and $\sum_{i=1}^{L} P_{b i}^{*}=\sum_{i=1}^{L} E_{b i}$; or $\sum_{i=1}^{L} P_{t i}^{*}+P_{b i}^{*}=\sum_{i=1}^{L} E_{s i}$.

Proof: We prove this statement by contradiction. Assume $\sum_{i=1}^{L} P_{t i}^{*}+P_{b i}^{*}<\sum_{i=1}^{L} E_{s i}$ and $\sum_{i=1}^{L} P_{t i}^{*}<\sum_{i=1}^{L} E_{t i}$. Now, we can increase $P_{t L}^{*}$ without violating the energy constraints 


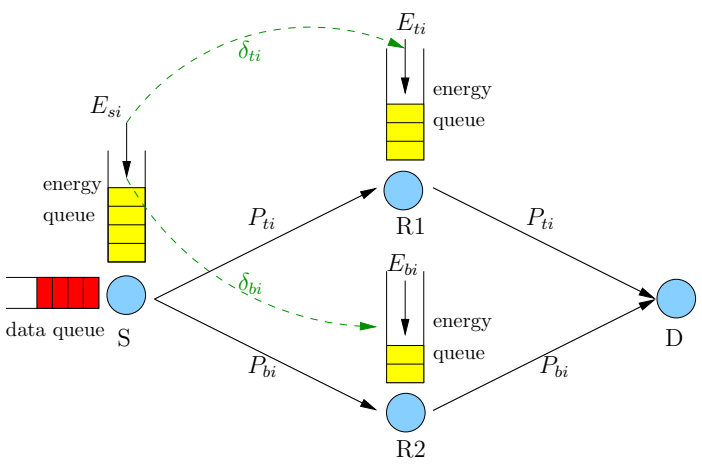

Fig. 2. Diamond network with strict delay requirements.

and improve the overall throughput which contradicts the optimality of $P_{t i}^{*}, P_{b i}^{*}$. Now, assume $\sum_{i=1}^{L} P_{t i}^{*}+P_{b i}^{*}<\sum_{i=1}^{L} E_{s i}$ and $\sum_{i=1}^{L} P_{b i}^{*}<\sum_{i=1}^{L} E_{b i}$. We can again increase $P_{b L}^{*}$ and improve the overall throughput contradicting the optimality. Therefore, the conditions stated in the lemma must hold.

Now we note that the problem in (11a)-(11d) is convex since the objective function is concave and the constraints are linear, and therefore can be solved by standard convex optimization techniques. The constraints in (11d) couple the energy causality constraints of the two relays. We propose the following decomposition for this problem. Let us define the function $f\left(\mathbf{P}_{t}\right)$ which is a maximization over $\mathbf{P}_{b}$ for fixed $\mathbf{P}_{t}$

$$
\begin{aligned}
& f\left(\mathbf{P}_{t}\right)=\max _{P_{b i} \geq 0} \sum_{i=1}^{L} \frac{1}{2} \log \left(1+P_{b i}\right) \\
& \text { s.t. }(11 \mathrm{c}), \sum_{i=1}^{k} P_{b i} \leq \sum_{i=1}^{k} E_{s i}-P_{t i}, \quad \forall k
\end{aligned}
$$

We have the following lemma.

Lemma $2 f\left(\mathbf{P}_{t}\right)$ is non-increasing and concave in $\mathbf{P}_{t}$.

Proof: Since increasing $\mathbf{P}_{t}$ can only shrink the feasible set, $f\left(\mathbf{P}_{t}\right)$ is non-increasing in $\mathbf{P}_{t}$. To prove the concavity: Let $\mathbf{P}_{t}$ and $\mathbf{Q}_{t}$ be two power vectors. Let $0 \leq \lambda \leq 1$ and $\bar{\lambda}=1-\lambda$. Let $\mathbf{P}$ be the solution of the problem $f\left(\mathbf{P}_{t}\right)$ and $\mathbf{Q}$ be the solution of the problem $f\left(\mathbf{Q}_{t}\right)$. $\mathbf{P}$ and $\mathbf{Q}$ exist and are unique due to convexity. The vector $\lambda \mathbf{P}+\bar{\lambda} \mathbf{Q}$ is feasible for the problem $f\left(\lambda \mathbf{P}_{t}+\bar{\lambda} \mathbf{Q}_{t}\right)$ as the constraints are linear. Then,

$$
\begin{aligned}
f\left(\lambda \mathbf{P}_{t}+\bar{\lambda} \mathbf{Q}_{t}\right) & \stackrel{(a)}{\geq} \sum_{i=1}^{L} \frac{1}{2} \log \left(1+\lambda P_{i}+\bar{\lambda} Q_{i}\right) \\
& \stackrel{(b)}{\geq} \sum_{i=1}^{L} \frac{\lambda}{2} \log \left(1+P_{i}\right)+\frac{\bar{\lambda}}{2} \log \left(1+Q_{i}\right) \\
& =\lambda \sum_{i=1}^{L} \frac{1}{2} \log \left(1+P_{i}\right)+\bar{\lambda} \sum_{i=1}^{L} \frac{1}{2} \log \left(1+Q_{i}\right) \\
& \stackrel{(c)}{=} \lambda f\left(\mathbf{P}_{t}\right)+\bar{\lambda} f\left(\mathbf{Q}_{t}\right)
\end{aligned}
$$

where $(a)$ follows because the maximum value of the problem can be no smaller than the objective value of any feasible point, (b) follows from the concavity of $\log$ and $(c)$ follows from the fact that $\mathbf{P}$ solves $f\left(\mathbf{P}_{t}\right)$ and $\mathbf{Q}$ solves $f\left(\mathbf{Q}_{t}\right)$.

The problem in (11a)-(11d) can equivalently be written as:

$$
\begin{array}{cc}
\max _{P_{t i} \geq 0} & f\left(\mathbf{P}_{t}\right)+\sum_{i=1}^{L} \frac{1}{2} \log \left(1+P_{t i}\right) \\
\text { s.t. } & (11 \mathrm{~b}), \sum_{i=1}^{k} P_{t i} \leq \sum_{i=1}^{k} E_{s i}, \quad \forall k
\end{array}
$$

where the second set of constraints is required to ensure the feasibility of problem (12). We solve the problem in (14) by separately solving the outer and inner maximization problems.

\section{A. Inner Maximization}

Here, we focus on the inner problem in (12) for a fixed $\mathbf{P}_{t}$. We combine the constraints in problem (12) to a single constraint by defining, $M_{k}=\min \left(\sum_{i=1}^{k} E_{b i}, \sum_{i=1}^{k} E_{s i}-P_{t i}\right)$ and $T_{k}=M_{k+1}-M_{k}$. Rewriting problem (12), we have

$$
\begin{aligned}
\max _{P_{b i} \geq 0} & \sum_{i=1}^{L} \frac{1}{2} \log \left(1+P_{b i}\right) \\
\text { s.t. } & \sum_{i=1}^{k} P_{b i} \leq \sum_{i=1}^{k} T_{i}, \quad \forall k
\end{aligned}
$$

This is the single-user throughput maximization problem with energy arrivals $T_{i}$ [5]-[7]. By Lemma 1 we must have $\sum_{i=1}^{L} P_{b i}^{*}=\sum_{i=1}^{L} T_{i}$ for $P_{t i}=P_{t i}^{*}$ which means that all of the energy will be utilized. This also follows from [5]-[7]. This problem can be solved by the geometric method in [5] or by the directional water-filling method in [7]. Once $P_{b i}^{*}$ are found, $f\left(\mathbf{P}_{t}\right)=\sum_{i=1}^{L} \frac{1}{2} \log \left(1+P_{b i}^{*}\right)$.

\section{B. Outer Maximization}

The outer maximization problem is the problem of finding the optimal $\mathbf{P}_{t}$ in (14). We combine the constraints in problem (14) to a single constraint by defining $N_{k}=$ $\min \left(\sum_{i=1}^{k} E_{t i}, \sum_{i=1}^{k} E_{s i}\right)$ and $Q_{k}=N_{k+1}-N_{k}$. Then, the problem in (14) can be written as:

$$
\begin{array}{ll}
\max _{P_{t i} \geq 0} & f\left(\mathbf{P}_{t}\right)+\sum_{i=1}^{L} \frac{1}{2} \log \left(1+P_{t i}\right) \\
\text { s.t. } & \sum_{i=1}^{k} P_{t i} \leq \sum_{i=1}^{k} Q_{i}, \quad \forall k
\end{array}
$$

This problem is convex since the constraints are linear and the objective function is concave by Lemma 2 and the concavity of the $\log$ function. By Lemma 2 the objective function here is not strictly increasing therefore all the available energy may not be utilized. This is especially the case when the top relay has sufficient energy but the source is energy deficient. As a result the excess energy in problem (16) has to be accounted for. To this end, we add an additional $(L+1)$ st slot to collect the excess energy. This additional slot does not contribute to 
the objective function. We use the following algorithm to solve this problem.

We utilize right permeable taps to account for the energy which is saved in the battery to be used in the future and an extra overflow slot at the very end to account for the extra energy that will not be utilized. We let each tap have a meter measuring the water that has already passed through it and we allow each tap to let the water flow back if an update in the allocation necessitates it. Any water that has flown to the overflow slot can also be called back through this process.

The water levels are obtained by allowing water flow from a single tap at a time. We start by filling the energy arrivals $Q_{i}$ in the slots with the taps closed and an empty overflow slot in the end. Water is allowed to flow forward if this flow increases the objective function in problem (16) and similarly water is allowed to flow backwards if this increases the objective function and the corresponding meter allows it. We note that if we go through all the possible taps sufficiently many times, our algorithm will converge to an optimal solution since the iterations increase the objective function monotonically while maintaining the feasibility at each iteration. Since the problem is convex, convergence to the optimal point is guaranteed.

\section{With ENERGY TRANSFER}

Now, we allow energy cooperation. With the possibility of energy transfer, we go back to the original problem in (10), and let $\boldsymbol{\delta}_{t} \geq \mathbf{0}, \boldsymbol{\delta}_{b} \geq \mathbf{0}$. We start with the following lemma.

Lemma 3 For optimal $\mathbf{P}_{t}^{*}, \mathbf{P}_{b}^{*}, \boldsymbol{\delta}_{t}^{*}, \boldsymbol{\delta}_{b}^{*}$, if for some slot $i, \delta_{t i}^{*}>$ $0, \delta_{b i}^{*}>0$ then $P_{t i}^{*}=P_{b i}^{*}$. Furthermore, if $\delta_{t i}^{*}>0, \delta_{b i}^{*}=0$ then $P_{t i}^{*} \leq P_{b i}^{*}$; and if $\delta_{t i}^{*}=0, \delta_{b i}^{*}>0$ then $P_{b i}^{*} \leq P_{t i}^{*}$.

Proof: Assume $\delta_{t i}^{*}>0, \delta_{b i}^{*}>0$ but $P_{t i}^{*} \neq P_{b i}^{*}$. First assume $P_{t i}^{*}>P_{b i}^{*}$. For $\epsilon>0$, define $\bar{P}_{t i}=P_{t i}^{*}-\epsilon, \bar{\delta}_{t i}=\delta_{t i}^{*}-\frac{\epsilon}{\alpha}, \bar{P}_{b i}=$ $P_{b i}^{*}+\epsilon, \bar{\delta}_{b i}=\delta_{b i}^{*}+\frac{\epsilon}{\alpha}$ while keeping all other variables the same. Now, we consider the modified policy $\left(\overline{\mathbf{P}}_{t}, \overline{\mathbf{P}}_{b}, \overline{\boldsymbol{\delta}}_{t}, \overline{\boldsymbol{\delta}}_{b}\right)$ and show that it is feasible. Since $\sum_{i=1}^{k} \bar{P}_{t i}-\alpha \bar{\delta}_{t i}=$ $\sum_{i=1}^{k} P_{t i}^{*}-\alpha \delta_{t i}^{*} \leq \sum_{i=1}^{k} E_{t i}, \forall k$ the constraints (9a) are satisfied. Similarly, $\sum_{i=1}^{k} \bar{P}_{b i}-\alpha \bar{\delta}_{b i}=\sum_{i=1}^{k} P_{b i}^{*}-\alpha \delta_{b i}^{*}, \forall k$ therefore (9b) are satisfied, and $\sum_{i=1}^{k} \bar{P}_{b i}+\bar{P}_{t i}-\bar{\delta}_{b i}-\bar{\delta}_{t i}=$ $\sum_{i=1}^{k} P_{b i}^{*}+P_{t i}^{*}-\delta_{t i}^{*}-\delta_{b i}^{*}, \forall k$ therefore (9c) are satisfied. By the concavity of the objective function, the modified policy achieves a higher throughput than the optimal policy which is a contradiction. Now assume $P_{b i}^{*}>P_{t i}^{*}$, and define $\bar{P}_{b i}=P_{b i}^{*}-\epsilon, \bar{\delta}_{b i}=\delta_{b i}^{*}-\frac{\epsilon}{\alpha}, \bar{P}_{t i}=P_{t i}^{*}+\epsilon, \bar{\delta}_{b i}=\delta_{t i}^{*}+\frac{\epsilon}{\alpha}$ to arrive at a similar contradiction. This proves the first statement. The second statement is proved by assuming $\delta_{t i}^{*}>0, \delta_{b i}^{*}=0$ but $P_{t i}^{*}>P_{b i}^{*}$ and modifying $\bar{P}_{t i}=P_{t i}^{*}-\epsilon, \bar{\delta}_{t i}=\delta_{t i}^{*}-\frac{\epsilon}{\alpha}, \bar{P}_{b i}=$ $P_{b i}^{*}+\epsilon, \bar{\delta}_{b i}=\frac{\epsilon}{\alpha}$ to reach a contradiction. The last statement follows from symmetry.

Lemma 3 reveals an interesting property of the optimal energy and information flow in the network. This lemma shows that if the source transfers non-zero amounts of energy to both relays, then the transmit powers of the source to both relays must be equal, implying that the source must send equal amounts of data to both relays. On the other hand, if the source sends energy to only one of the relays, then the transmit power of the source to that relay must be less. These together imply that if the source sends more energy to a relay then it sends less data. This shows how energy and data must flow together optimally in the network, and also reveals an interesting tradeoff between the amounts of information and energy transfers.

Next lemma shows that the class of procrastinating policies in [23] is optimal in our setting also. This means that the energies must be transferred only when they will be immediately used. That is no transferred energy should be saved and used in the future slots at the energy receiving node.

Lemma 4 Define a policy as procrastinating if it satisfies $P_{t k} \geq \alpha \delta_{t k}$ and $P_{b k} \geq \alpha \delta_{b k}, \forall k$. There exists a procrastinating policy that solves problem (10).

Proof: We prove that any feasible policy can be made procrastinating without modifying the sum-rate. Assume a feasible $\left(\mathbf{P}_{t}, \mathbf{P}_{b}, \boldsymbol{\delta}_{t}, \boldsymbol{\delta}_{b}\right)$ is not procrastinating and there exists at least one $k$ and $j$ such that $P_{t k}<\alpha \delta_{t k}$ or $P_{b j}<\alpha \delta_{b j}$. First, we focus on the top relay. Choose the smallest such $k$, define $\bar{\delta}_{t k}=\delta_{t k}-\epsilon$ and $\bar{\delta}_{t(k+1)}=\delta_{t(k)}+\epsilon$, where $\epsilon$ is chosen to satisfy $P_{t k}=\alpha \bar{\delta}_{t k}$, while keeping all other variables the same. If $k=L$, only modify $\bar{\delta}_{t L}=\bar{\delta}_{t L}-\epsilon$. Now, we show that the modified policy $\left(\mathbf{P}_{t}, \mathbf{P}_{b}, \overline{\boldsymbol{\delta}}_{t}, \boldsymbol{\delta}_{b}\right)$ is feasible. We have $\sum_{i=1}^{k} P_{t i}-\alpha \bar{\delta}_{t i}=\sum_{i=1}^{k-1} P_{t i}-\alpha \bar{\delta}_{t i}=\sum_{i=1}^{k-1} P_{t i}-\alpha \delta_{t i} \leq$ $\sum_{i=1}^{k-1} E_{t i} \leq \sum_{i=1}^{k} E_{t i}$. Therefore, (9a) is satisfied at slot $\bar{k}$. Since $\sum_{i=1}^{p} \bar{\delta}_{t i}=\sum_{i=1}^{p} \delta_{t k}$ for $p>k$, constraints in (9a) for slots $p>k$ are identical and satisfied. Constraints in (9c) are satisfied because at slot $k, \bar{\delta}_{t k}<\delta_{t k}$, which can only extend the feasible region and the constraints at slots $p>k$ are identical. This modification does not change $\mathbf{P}_{t}$ and achieves the same sum-rate, however, the time slot $k$ that prevents the policy from being non-procrastinating is strictly increased. By repeatedly applying this procedure, the policy $\mathbf{P}_{t}$ is made procrastinating. Similar modification to the bottom relay makes the policy $\mathbf{P}_{b}$ procrastinating, and completes the proof of the lemma.

Let us define $\bar{P}_{t i}=P_{t i}-\alpha \delta_{t i}$ and $\bar{P}_{b i}=P_{b i}-\alpha \delta_{b i}$. A procrastinating policy satisfies $\bar{P}_{t i} \geq 0$ and $\bar{P}_{b i} \geq 0$.We rewrite the problem in (10) in terms of these new variables as:

$$
\begin{aligned}
\max _{\substack{\overline{\mathbf{P}}_{t}, \overline{\mathbf{P}}_{b} \geq \mathbf{0} \\
\boldsymbol{\delta}_{t}, \boldsymbol{\delta}_{b} \geq \mathbf{0}}} & \sum_{i=1}^{L} \frac{1}{2} \log \left(1+\bar{P}_{t i}+\alpha \delta_{t i}\right)+\frac{1}{2} \log \left(1+\bar{P}_{b i}+\alpha \delta_{b i}\right) \\
\text { s.t. } & \sum_{i=1}^{k} \bar{P}_{t i} \leq \sum_{i=1}^{k} E_{t i}, \quad \forall k \\
& \sum_{i=1}^{k} \bar{P}_{b i} \leq \sum_{i=1}^{k} E_{b i}, \quad \forall k \\
& \sum_{i=1}^{k} \bar{P}_{t i}+\bar{P}_{b i} \leq \sum_{i=1}^{k} E_{s i}-(17 \mathrm{a})
\end{aligned}
$$


The only energy causality constraints on $\left(\boldsymbol{\delta}_{t}, \boldsymbol{\delta}_{b}\right)$ are (17d). Let us define $g\left(\overline{\mathbf{P}}_{t}, \overline{\mathbf{P}}_{b}\right)$ as

$$
\begin{aligned}
g\left(\overline{\mathbf{P}}_{t}, \overline{\mathbf{P}}_{b}\right)=\max _{\boldsymbol{\delta}_{t}, \boldsymbol{\delta}_{b} \geq \mathbf{0}} & \sum_{i=1}^{L} \frac{1}{2} \log \left(1+\bar{P}_{t i}+\alpha \delta_{t i}\right) \\
& +\frac{1}{2} \log \left(1+\bar{P}_{b i}+\alpha \delta_{b i}\right) \\
\text { s.t. } \quad & (17 \mathrm{~d})
\end{aligned}
$$

Now, the problem (17a)-(17d) can equivalently be written as:

$$
\begin{array}{rl}
\max _{\overline{\mathbf{P}}_{t}, \overline{\mathbf{P}}_{b} \geq \mathbf{0}} & g\left(\overline{\mathbf{P}}_{t}, \overline{\mathbf{P}}_{b}\right) \\
\text { s.t. } & (17 \mathrm{~b}),(17 \mathrm{c}), \sum_{i=1}^{k} \bar{P}_{t i}+\bar{P}_{b i} \leq \sum_{i=1}^{k} E_{s i} \quad \forall k
\end{array}
$$

where the last set of constraints ensure the feasibility of (18).

\section{A. Inner Maximization}

Here, we solve (18) for fixed $\overline{\mathbf{P}}_{t}, \overline{\mathbf{P}}_{b}$. We rewrite (17d) more conveniently as $\sum_{i=1}^{k} \alpha\left(\delta_{t i}+\delta_{b i}\right) \leq \sum_{i=1}^{k} Z_{i}$ where $Z_{i} \triangleq$ $\alpha\left(E_{s i}-\bar{P}_{t i}-\bar{P}_{b i}\right) /(1+\alpha)$. The solution to this problem can be found by water-filling the energy levels $Z_{i}$ on top of two users with the base levels $\bar{P}_{t i}$ and $\bar{P}_{b i}$, respectively. Then, $g\left(\overline{\mathbf{P}}_{t}, \overline{\mathbf{P}}_{b}\right)$ is found by plugging the optimal energy transfer values in the objective function of (18). Using a similar argument as in Lemma 2, it can be shown that $g\left(\overline{\mathbf{P}}_{t}, \overline{\mathbf{P}}_{b}\right)$ is jointly concave in $\left(\overline{\mathbf{P}}_{t}, \overline{\mathbf{P}}_{b}\right)$.

\section{B. Outer Maximization}

Here, we solve (19) by finding the optimal $\left(\overline{\mathbf{P}}_{t}, \overline{\mathbf{P}}_{b}\right)$. The constraints of problem (19) are equivalent to that of problem (11a)-(11d) but the objective function is modified from the sum-rate to $g\left(\overline{\mathbf{P}}_{t}, \overline{\mathbf{P}}_{b}\right)$ which is concave. By using procrastinating policies, we have reduced the problem with energy cooperation to a problem without energy cooperation which has a modified objective function. Since $g\left(\overline{\mathbf{P}}_{t}, \overline{\mathbf{P}}_{b}\right)$ is concave, we can use the algorithm specified in Section III-B.

\section{Numerical ExAmple}

We provide a numerical example over 3 slots to illustrate our results. Let $\mathbf{E}_{s}=[5,3,10], \mathbf{E}_{t}=[2,6,0]$ and $\mathbf{E}_{b}=$ $[0.5,4,2]$ be harvested by the source, top relay and bottom relay, respectively, and $\alpha=0.5$. In the case of no energy cooperation, the optimal solution is $\mathbf{P}_{t}^{*}=[2,2.75,3.25]$ and $\mathbf{P}_{b}^{*}=[0.5,2.75,3.25]$ with a sum-rate of 7.04 . In the first slot, each relay exhausts its own energy and in the remaining slots the source energy is divided equally among the relays. In time, the power levels are equalized to the extent possible by the relay energy causality constraints. Energy is left at the source, but both relay energies are used up in agreement with Lemma 1. With the possibility of energy transfer, the optimal values converge to $\mathbf{P}_{t}^{*}=[2,2.23,4.18], \mathbf{P}_{b}^{*}=$ $[0.84,2.23,4.18], \boldsymbol{\delta}_{t}^{*}=[0,0,0.82], \boldsymbol{\delta}_{b}^{*}=[0.69,0,0.82]$, and a sum-rate of 7.34 is achieved. We see that $\delta_{t 1}^{*}=0, \delta_{b 1}^{*}>$ $0, P_{b 1}^{*} \leq P_{t 1}^{*}$ and $\delta_{t 3}^{*}>0, \delta_{b 3}^{*}>0, P_{b 3}^{*}=P_{t 3}^{*}$ in agreement with Lemma 3 . The resulting policy is procrastinating in agreement with Lemma 4.

\section{CONCLUSIONS}

We studied the energy harvesting diamond channel with signal and energy cooperation, and solved the end-to-end throughput maximization problem by convex decomposition. For the case with energy cooperation, we determined the jointly optimum information and energy flow in the network.

\section{REFERENCES}

[1] B. Schein and R. Gallager. The Gaussian parallel relay network. In IEEE ISIT, June 2000.

[2] A. Sendonaris, E. Erkip, and B. Aazhang. User cooperation diversity. Part I. System description. IEEE Trans. on Comm., 51(11):1927-1938, November 2003.

[3] A. Kurs, A. Karalis, R. Moffatt, J.D. Joannopoulos, P. Fisher, and M Soljacic. Wireless power transfer via strongly coupled magnetic resonances. Science, 317:83-86, July 2007.

[4] B. Gurakan, O. Ozel, J. Yang, and S. Ulukus. Energy cooperation in energy harvesting communications. IEEE Trans. Comm., 61(12):48844898, December 2013.

[5] J. Yang and S. Ulukus. Optimal packet scheduling in an energy harvesting communication system. IEEE Trans. Comm., 60(1):220-230, January 2012.

[6] K. Tutuncuoglu and A. Yener. Optimum transmission policies for battery limited energy harvesting nodes. IEEE Trans. Wireless Comm., 11(3):1180-1189, March 2012.

[7] O. Ozel, K. Tutuncuoglu, J. Yang, S. Ulukus, and A. Yener. Transmission with energy harvesting nodes in fading wireless channels: Optimal policies. IEEE JSAC, 29(8):1732-1743, September 2011.

[8] J. Yang, O. Ozel, and S. Ulukus. Broadcasting with an energy harvesting rechargeable transmitter. IEEE Trans. Wireless Comm., 11(2):571-583, February 2012.

[9] M. A. Antepli, E. Uysal-Biyikoglu, and H. Erkal. Optimal packet scheduling on an energy harvesting broadcast link. IEEE JSAC, 29(8):1721-1731, September 2011.

[10] O. Ozel, J. Yang, and S. Ulukus. Optimal broadcast scheduling for an energy harvesting rechargeable transmitter with a finite capacity battery. IEEE Trans. Wireless Comm., 11(6):2193-2203, June 2012.

[11] J. Yang and S. Ulukus. Optimal packet scheduling in a multiple access channel with energy harvesting transmitters. Journal of Comm. and Netw., 14:140-150, April 2012.

[12] K. Tutuncuoglu and A. Yener. Sum-rate optimal power policies for energy harvesting transmitters in an interference channel. Journal of Comm. and Netw., 14(2):151-161, April 2012.

[13] D. Gunduz and B. Devillers. Two-hop communication with energy harvesting. In IEEE CAMSAP, December 2011.

[14] C. Huang, R. Zhang, and S. Cui. Throughput maximization for the Gaussian relay channel with energy harvesting constraints. IEEE JSAC, 31:1469-1479, August 2013.

[15] O. Orhan and E. Erkip. Optimal transmission policies for energy harvesting two-hop networks. In CISS, March 2012.

[16] Y. Luo, J. Zhang, and K. B. Letaief. Optimal scheduling and power allocation for two-hop energy harvesting communication systems. IEEE Trans. Wireless Comm., 12(9):4729-4741, September 2013.

[17] B. Devillers and D. Gunduz. A general framework for the optimization of energy harvesting communication systems with battery imperfections. Journal of Comm. and Netw., 14(2):130-139, April 2012.

[18] K. Tutuncuoglu and A. Yener. Communicating using an energy harvesting transmitter: Optimum policies under energy storage losses. IEEE Trans. Wireless Comm., submitted. Also available at [arXiv:1208.6273].

[19] O. Orhan, D. Gunduz, and E. Erkip. Throughput maximization for an energy harvesting communication system with processing cost. In IEEE ITW, September 2012.

[20] J. Xu and R. Zhang. Throughput optimal policies for energy harvesting wireless transmitters with non-ideal circuit power. IEEE JSAC, 32(2):322-332, February 2014.

[21] O. Orhan and E. Erkip. Throughput maximization for energy harvesting two-hop networks. In IEEE ISIT, July 2013.

[22] K. Tutuncuoglu and A. Yener. Multiple access and two-way channels with energy harvesting and bidirectional energy cooperation. In UCSD ITA Workshop, February 2013.

[23] K. Tutuncuoglu and A. Yener. Cooperative energy harvesting communications with relaying and energy sharing. In IEEE ITW, Sep. 2013. 